\title{
Adaptive Weighted Morphology Detection Algorithm of Plane Object in Docking Guidance System
}

\author{
Guo Yan-Ying1, Yang Guo-Qing1 and Jiang Li-Hui 2 \\ 1 Nanjing University of Aeronautics and Astronautics \\ 2 Tianjin key lab for advanced signal processing Civil \\ Aviation University of China \\ China
}

\section{Introduction}

Aerodrome docking auto-guidance system is no-manual work guidance plane from taxiway to gate position and nicety anchor process. Aerodrome docking auto-guidance system can ensure plane safe nice anchor, and make corridor bridge meet plane. At present there are no departments of docking auto-guidance system in China. Based on vision detect method of aerodrome docking auto-guidance system refer to literatures a flat lot. In the paper, importantly study image pretreatment algorithm based on vision detection of aerodrome docking auto-guidance system, process and so on. Docking system chart is figure 1.

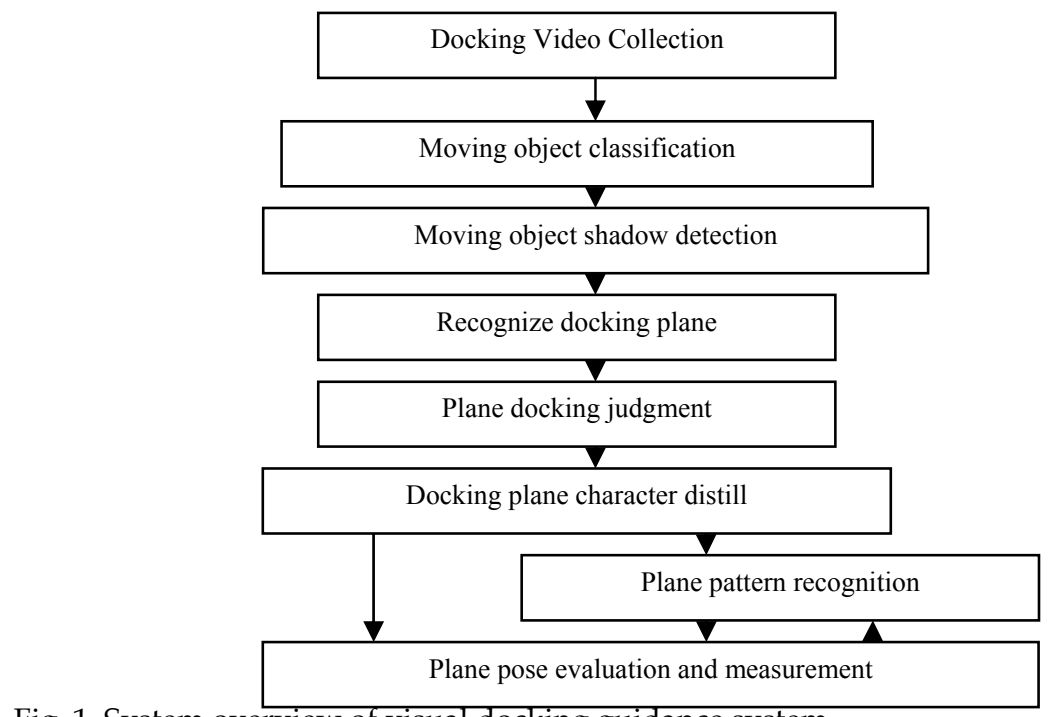

Fig. 1. System overview of visual docking guidance system 
The edge of image embodies a great deal of information, is the important attribute to obtain image features during image identification. Edge detection could draw the outline of target object to reach object identification. Essentially, image edge is the discontinuous reflection of part of image; it indicates one ending of one field and starting of another field. Effect of edge detection influenced directly accomplishment of after work (Lima, P.; Bonarini, A. \& Mataric, M. (2004)). Edges can also be defined as where gradient of image intensity function reaches its local maximum. In other words, edge points are points in the image where pixel brightness changes drastically. Typically, edge points are associated with the boundaries of objects in the image and edge detection can also be used for region segmentation and feature extraction. Edge detection methods can be classified into directional and nondirectional or gradient-based operators (M. Pesaresi and J. A. Benediktsson2001, Heath M., Sarkar S., Sanocki T., and Bowyer K 1998, Ziou D. and Tabbone S 1997). Directional operators use two masks and two convolutions. While non-directional use single mask and convolution but they are sensitive to noise due to gradient nature of the operators. In near year, classical popular gradient-based edge detection algorithms were experimented on the binary images There is many methods of edge detection, traditional method adopt edge detection operator to solve two dimensional real function, then selecting proper threshold to extract edge, these classical edge detection operator solution mainly is Roberts, Prewitt, Sobel and Canny operator, etc. When image is in the condition without noise interruption, these operators could provide perfect edge (M. K. Kundu, B. B. Chaudhuri and D. Dutta Majumder, 1991). But, actually noise existed and presented abrupt change between noise and edge, and high frequency information in frequency field. So it is difficult for edge detection. Under such circumstance, classical operator couldn't extract edge well, firstly filter the noise, and then detect edge. This could not only bring extra calculation amount, but wipe off the faint edge (W. K. Pratt, 1991). Mathematical morphology (MM) base on group algorithm, characteristic of non-linearity, and it not only presents image group features to well detect image edge, but also satisfies real-time request. What's more? Based on edge detection, through changing the shape and size of structure element extract image edge, in order to overcome the influence of noise (SONG J, DELPE J 1990,(50)). MM has achieved the status of a powerful tool in the design of edge detection and nonlinear filters for signal/image processing (Lima, P.; Bonarini, A. \& Mataric, M. 2004, M. Pesaresi and J. A. Benediktsson2001, Heath M., Sarkar S., Sanocki T., and Bowyer K 1998) .

In this paper, we present a new edge detection algorithm based on adaptive weighted morphological operations. The primary objective of the adaptive weighted morphological grads operations in the proposed algorithm is to generate the high connectively edge features of the image. Then apply edge to select structure element, If the edge direction exists, a big weight factor is put; if doesn't exist, a small weight factor is put. Thus we can achieve an intensified edge detector.

This paper is organized as follows: Section 2 introduces the weighted morphological operations. And proposes adaptive weighted morphological edge detection used in the proposed algorithm. Section3 presents comprehensive comparison results between the proposed algorithms with other existing methods. The conclusions and discussions are provided in the last section. 


\section{Adaptive Weighted Morphological Algorithm}

\subsection{Basic Theory of Mathematical Morphology and Conventional Edge Conventional Edge Detection Operators}

Mathematical Morphology is a framework based exclusively on set theory, which has found great success and applicability in digital image processing. The mathematical foundation of mathematical morphology comes from Minkowsy's set operations, through which its two basic operators are defined: dilation and erosion. Dilation can be stated in a simplified, intuitionist manner, as adding pixels to an image, or enlarging it. On the other hand, erosion can be stated in a similar manner as taking pixels away from an image, or shrinking it. The combination and interaction of these two operators, dilation and erosion, give rise to other two operations of singular importance in mathematical morphology: opening and closing. On one hand, opening consists of applying an erosion followed by a dilation to a set, with the same structuring element. As a result, small holes are enlarged. On the other hand, closing consists on applying a dilation followed by an erosion to a set, with the same structuring element. As a result, small holes are closed.

The objective of the present paper is to state mathematical morphology basic operations in terms of cellular automata, with the goal of enlightening the framework of mathematical morphology with the theory of cellular automata. Mathematical Morphology (MM) is a new science based on strict math theory basis, taking set theory as basis ,analyzing and understanding the digital image, which is also a good tool of geometry morphologic analysis and description; having be a new theory and method under digital image management area, producing great influence on digital image management theory and technology. In digital image, edge contains a great deal of valuable information, and it can reflect character of object, so it has an important intention about image analysis and image filtering. Math Morphology is the tool for analyzing the image based on structure element. Basic ideas are to measure and extract corresponding shape using structure element which have specific figuration to reach the image of analyzing and identification.

There are 4 basic algorithms in MM: dilation, erosion, opening and closing algorithm, they are of own characteristic in binary image and grey-scale image. These basic algorithms also induce and combine various math morphological algorithms (SONG J, DELPE J 1990).

A. Dilation and Erosion

Dilation and erosion operations are fundamental to morphological processing. In fact, many of the morphological algorithms are based on these two primitive operations.

1) Dilation

With $\mathrm{A}$ and $\mathrm{B}$ as sets in $Z^{2}$, the dilation of $\mathrm{A}$ by $\mathrm{B}$, denoted $A \oplus B$, is defined as

$$
A \oplus B=\left\{z \mid(\hat{B})_{z} \bigcap A \neq \varnothing\right\}
$$

Equation (1) is based on obtaining the reflection of B about its origin and shifting this reflection by $z$, such that $\hat{B}$ and A overlap by at least one element. Based on this interpretation, equation (1) may be rewritten as 


$$
A \oplus B=\left\{z \mid\left[(\hat{B})_{z} \bigcap A\right] \subseteq A\right\}
$$

Set B is commonly referred to as the structuring element in dilation, as well as below morphological operations.

2) Erosion

For sets $\mathrm{A}$ and $\mathrm{B}$ in the $Z^{2}$, the erosion of $\mathrm{A}$ by $\mathrm{B}$, denoted $A \otimes B$, is defined as

$$
A \otimes B=\left\{z \mid(B)_{z} \subseteq A\right\}
$$

In a word, the equation (3) indicates that the erosion of $\mathrm{A}$ by B is the set of all points $z$ such that $\mathrm{B}$, translated by $Z$, is contained in $\mathrm{A}$.

Dilation and erosion are duals of each other with respect to set complementation and reflection. That is,

$$
(A \otimes B)^{c}=A^{c} \oplus \hat{B}
$$

\section{B. Opening and Closing}

As can be seen from the equation (1) and (3), dilation expands an image and erosion shrinks it. Two other important morphological operations are introduced below: opening and closing. Opening generally smoothes the contour of an object, breaks narrow isthmuses, and eliminates thin protrusion. Closing also tends to smooth sections of contours but, as opposed to opening, it generally fuses narrow breaks and long thin gulfs, eliminates small holes, and fills gaps in the contour.

The opening of the set $\mathrm{A}$ by structuring element $\mathrm{B}$, denoted $A \circ B$, is defined as

$$
A \circ B=(A \otimes B) \oplus B
$$

Thus, the opening by $\mathrm{B}$ is the erosion of $\mathrm{A}$ by $\mathrm{B}$, followed by dilation of the result by $\mathrm{B}$.

Similarly, the closing of set $\mathrm{A}$ by structuring element $\mathrm{B}$, denoted $A \bullet B$, is defined as

$$
A \bullet B=(A \oplus B) \otimes B
$$

Which, in words, says that the closing of by B is simply the dilation of A by B, followed by the erosion of the result by $\mathrm{B}$.

Use the basic operations of dilation, erosion, opening and closing to develop several basic gray-scale morphological algorithms, especially, for edge extraction via the morphological operations. Throughout the discussions that follow, digital image functions of the form $f(x, y)$ and $b(x, y)$ are dealt with, where $f(x, y)$ is the input image and $b(x, y)$ is a 
structuring element, itself a sub-image function. The assumption is that these functions that assign a gray-level value which is a real number from the set of real numbers.

A. Gray-Scale Dilation

Gray-scale dilation of $f_{\text {by }} b$, denoted $f \oplus b$, is defined as

$$
(f \oplus b)(s, t)=\max \left\{f(s-x, t-y)+b(x, y) \mid(s-x),(t-y) \in D_{f} ;(x, y) \in D_{b}\right\}
$$

Where $D_{f}$ and $D_{b}$ are the domains of $f$ and $b$, respectively. $f$ and $b$ are functions rather than sets.

The condition that $(s-x)$ and $(t-y)$ have to be in the domain of $f$, and $x$ and $y$ have to be in the domain of $b$, is analogous to the condition in the binary definition of dilation, where the two sets have to overlap by at least one element.

B. Gray-scale Erosion

Gray-scale erosion, denoted $f \otimes b$, is defined as

$$
(f \otimes b)(s, t)=\min \left\{f(s+x, t+y)-b(x, y) \mid(s+x),(t+y) \in D_{f} ;(x, y) \in D_{b}\right\}
$$

Where $D_{f}$ and $D_{b}$ are the domains of $f_{\text {and }} b$, respectively. The condition that $(s+x)$

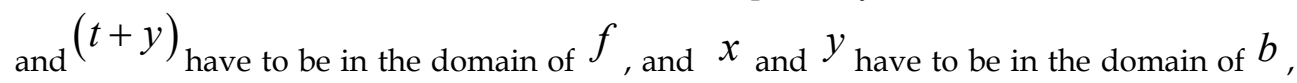
is analogous to the condition in the binary definition of erosion, where the structuring element has to be completely contained by the set being eroded.

C. Gray-scale Opening and Closing

The expressions for opening closing of gray-scale images have the same form as their binary counterparts. The opening of image $f$ by sub-image (structuring element) $b$, denoted $f \circ b$, is

$$
f \circ b=(f \otimes b) \oplus b
$$

Opening is the erosion of $f_{\text {by }} b$, followed by a dilation of the result by $b$.

Similarly, the closing of $f_{\text {by }} b$, denoted $f \bullet b$, is

$$
f \bullet b=(f \oplus b) \otimes b
$$

The opening and closing for gray-scale images are duals with respect to complementation and reflection. That is, 


$$
(f \bullet b)^{c}=f^{c} \circ \hat{b}
$$

Because $f^{c}=-f(x, y)$, equation (11) can be written also as

$$
-(f \cdot b)^{c}=(-f \circ \hat{b})
$$

Opening and closing of images have a simple geometric interpretation. Suppose that we view an image function $f(x, y)$ in 3-D perspective, with $x$ and $y$ axes being the usual spatial coordinates and the third axis being gray-level values. In this representation, the image appears as a discrete surface whose value at any point $(x, y)$ is the value of $f$ at those coordinates. Suppose that we open $f_{\text {by a spherical structuring element }} b$, viewing this element as a "rolling ball". Then the mechanics of opening $f_{\text {by }} b$ may be interpreted geometrically as the process of pushing the ball against the underside of the surface, while at the same time rolling it so that the entire underside of the surface of the highest points reached by any part of the sphere as it slides over the entire undersurface of $f$.

C. Conventional Edge Detection Operators in Edge Detection

1) Robert Operator

Robert operator is an easy edge detection operator using local difference operator to detect edge. It is an effective operator for the image with steep low noise. Robert operator can be expressed as the form of two as shown in Fig. 2. Two masks are used to calculate $\nabla_{x} f$ and $\nabla_{y} f_{\text {respectively. }}$

\begin{tabular}{|c|c|}
\hline 1 & 0 \\
\hline 0 & -1 \\
\hline
\end{tabular} \begin{tabular}{|c|c|}
\hline 0 & 1 \\
\hline-1 & 0 \\
\hline
\end{tabular}

Fig.2. Two masks of Robert operator in $x$ and $y$ direction.

2) Sobel Operator

Sobel operator is used to detect edge in the form of filter operator. Sobel operator can be expressed as the form of two masks as shown in Fig. 3. They are used to calculate the convolution of each pixel. The first mask has strong effect on vertical edge. The second mask has strong effect on horizontal edge. The max values of two convolutions are regarded as the output points. The result is an edge magnitude image. 


\begin{tabular}{|c|l|l|}
\hline-1 & 0 & 1 \\
\hline-2 & 0 & 2 \\
\hline-1 & 0 & 1 \\
\hline
\end{tabular}

\begin{tabular}{|c|c|c|}
\hline 1 & 2 & 1 \\
\hline 0 & 0 & 0 \\
\hline-1 & -2 & -1 \\
\hline
\end{tabular}

Fig.3. Two masks of Sobel operator in $x$ and $y$ direction.

3) Prewitt Operator

The same as Sobel operator each pixel in the image is calculated the convolution by using two masks shown in Fig. 4 and gain the max values in different directions. The result is also an edge magnitude image.

\begin{tabular}{|c|l|l|}
\hline-1 & 0 & 1 \\
\hline-1 & 0 & 1 \\
\hline-1 & 0 & 1 \\
\hline
\end{tabular}

\begin{tabular}{|c|c|c|}
\hline 1 & 1 & 1 \\
\hline 0 & 0 & 0 \\
\hline-1 & -1 & -1 \\
\hline
\end{tabular}

Fig.4. Two masks of Prewitt operator in $x$ and $y$ direction.

In the process of digital image management, difference gradient operator often combines with threshold technique for image edge detection. In the same condition of difference gradient operator, morphological gradient operator can also combine with threshold for using to complete edge detection. In the process of which, if gradient value of one place is large, then shows that change was rapid at this point of image, so edge is likely to pass through. These gradients can be give out as digital difference format. Three morphological gradient operators were defined.

\subsection{Weighted Morphological Operators}

We define weighted erosion (WER) and dilation (WDI) as (1) (2).

$$
\begin{aligned}
& W E R(k, l)=\min _{u, v}\{X(k+u, l+v) / B(u, v)\} \\
& W E R(k, l)=\min _{u, v}\{X(k+u, l+v) / B(u, v)\}
\end{aligned}
$$

There is $\mathrm{X}$ original image, B structuring element. The other operators, such as weighted opening (WOP) and closing (WCL), are simply cascades of weighted erosion and dilation which can be described as $\operatorname{WOP}(X)=W D I(W E R(X))$ and $W C L(X)=W E R(W D I(X))$, respectively. Weighted open-closing (WOPCL) and close-opening (WCLOP) are denoted as $W O P C L(X)=W C L(W O P(X))$ and $W C L O P(X)=W O P(W C L(X))$, respectively. The structuring element $B$ has a normalized weight factor and its elements are calculated such that the edge directional point's weight is 1 and the farthest point's weight is assigned a weight factor $\omega>1$, leading to an emphasis on the effect of the edge directional point and a reduction of the effect of the neighborhood points. The rest of the weights are calculated based on an increment $\Delta \omega=(\omega-1) / d$, where $d$ is the distance between the edge directional point and the farthest point from the edge directional point. In the vertical and horizontal directions, the weight 
decrease by $\Delta \omega$, each step starting from the edge directional point. For example, for an SE of size $3 \times 3$ with its edge direction at the horizontal direction, if $\omega_{1}=3$, the SE will look like $B_{1}$, where $\Delta \omega=2$, and for $\omega_{2}=3$, for the same edge direction point (underlined) at the oblique 45 angle, it will change to $B_{2}$, where $\Delta \omega=1$.

$$
B_{1}=\left[\begin{array}{ccc}
-1 & -1 & -1 \\
1 & \underline{1} & \underline{1} \\
-1 & -1 & -1
\end{array}\right] \quad B_{2}=\left[\begin{array}{ccc}
\underline{1} & 0 & -1 \\
0 & \underline{1} & 0 \\
-1 & 0 & \underline{1}
\end{array}\right]
$$

\subsection{Adaptive Weighted Morphological Edge Detection}

In mathematical morphological operations, there are always two sets involved: The shape of an image is determined by the values that the signal takes on. The shape information of the image is extracted by using a structuring element to operate on the image. So morphological image processing lies on morphological operations combination and structuring elements. In case operations' mode is selected, relevant result is ascertained by structuring elements. Yet designing effective structuring elements is a difficult task. In this paper, a new algorithm based adaptive weighted morphological operations is proposed, adaptive select structuring element to extract edge. Morphological grads operator $(X \oplus B)-(X \Theta B)$ can reinforce comparatively speculate grey transition region in images. So the operator is applied to detect edge in the paper and simultaneity adaptive select structuring element to extract edge.

The weighted morphological operators emphasize the edge direction points of window effect. Therefore an adaptive weighted morphological transformation algorithm is proposed. If pixel in the edge of window is edge, a big weight factor is put; if it doesn't exist, a small weight factor is put. Thus the probability of the edge detection is improved by morphological operations. So the first step is looking for edge in this paper. Circumrotate cover edge search method is adopted. SE apply rectangle cover figure.1, let it circumrotation goes around the center edge of SE. Then ensure position of grey variance maximum in rectangle cover, so we may employ variance as domanial estimate. By way of preferably extract edge of image, apply figure formal cover mode.

The structuring element is confirmed, then adopt adaptive weighted matrix to detect image edge. The structuring element $B$ has a normalized weight factor and its elements are calculated such that the edge directional point's weight is 1 and the farthest point's weight is assigned a weight factor $0<\omega<1$, leading to an emphasis on the effect of the edge directional point and a reduction of the effect of the neighborhood points. The rest of the weights are calculated based on an increment $\Delta \omega=(1-\omega) / d$, where $d$ is the distance between the edge directional point and the farthest point from the edge directional point. In the vertical and horizontal directions, the weight decrease by $\Delta \omega$, each step starting from the edge directional point.

Finally, whether or not edge pixels exist is judged by selecting weight SE can let non-edge pixels give small weight factor, yet let edge pixels give big weight factor. When the 
structuring elements is toned or restrained, edge is given prominence by morphological swell operation; edge is given weaken by morphological erode operation. So edge is much more extruded. At the same time some of the small detail edges are more distinct.

\section{Simulation Experiments and Results}

In this paper, the Lena image and Docking plane image are used in this experiment to process edge detection. Several popular gradient-based edge detection algorithms were experimented on the images, including Sobel, Laplacian of Gaussian and so on. The original image, as shown in Fig.5, Laplacian of Gaussian Edge Detector image, as shown in Fig.6, Sobel edge detector image, as shown in Fig.7, and the proposed method image, as shown in Fig.8.Edge detect image of docking plane is shown in Fig.9.

As can be seen from the images, Sobel edge detector is highly subjected to detection of false edges. This is probably due to the high threshold value used to detect weak edges in the Sobel detector. The most common error that occurred in four detectors (Sobel and LoG) tested are missing true edges. This is especially obvious in Laplacian of Gaussian detector probably due to the smoothing operation prior to edge detection. Laplacian of Gaussian detector also produces a small degree of disconnected edges especially in complex shapes such as map in Lena. The proposed method can not only primely extract detail edge, but also superbly preserve integer effect.

The proposed method is based on whether or not edge pixels exist is judged by selecting weight SE can let non-edge pixels give small weight factor, yet let edge pixels give big weight factor. The method can generate the high connectively edge features by other method effectively. Image edges are clear and false edges almost not exist. At the same time some of the small details edges are more distinct. Therefore, the proposed method is advantage of their algorithm comparing with other algorithm.

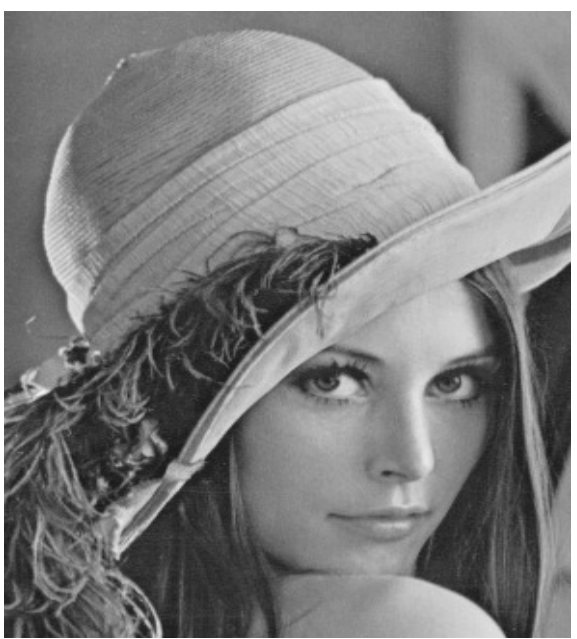

Fig. 5. Original image

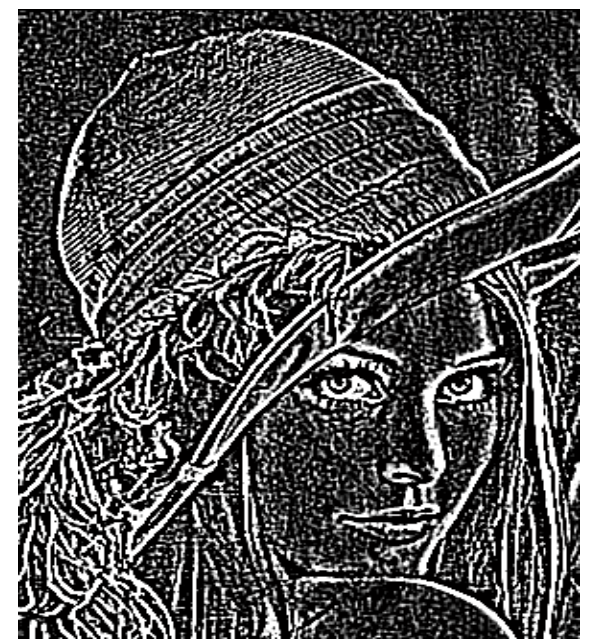

Fig. 6. LOG edge image 


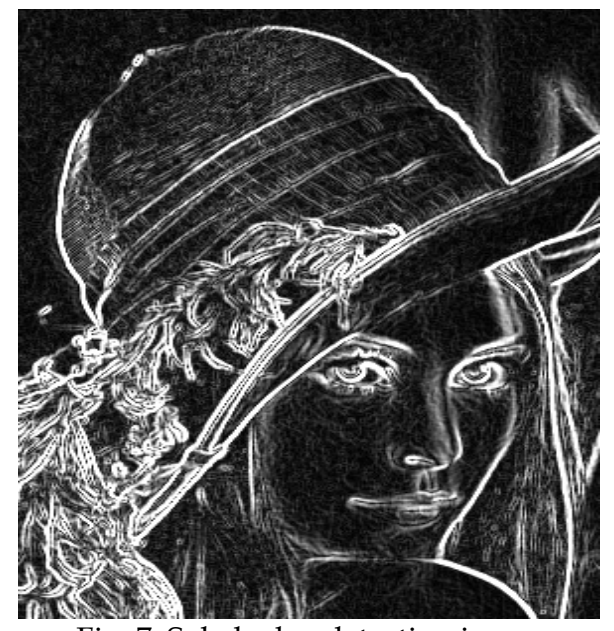

Fig. 7. Sobel edge detection image

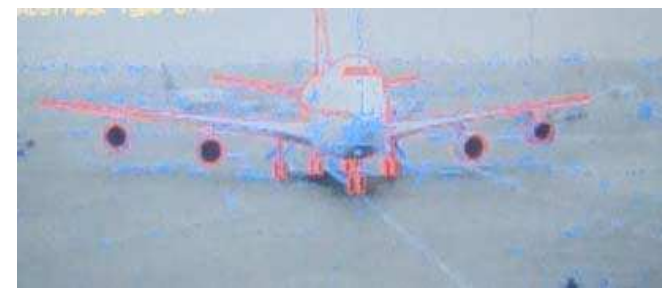

Fig.9. (a)

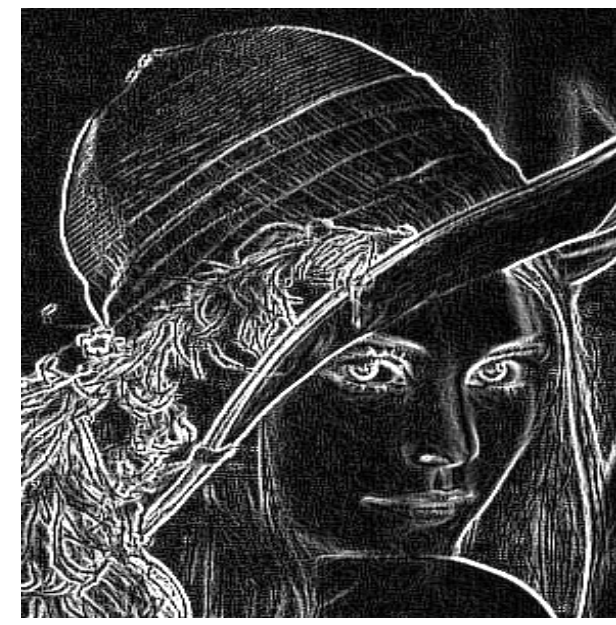

Fig. 8. the proposed method image

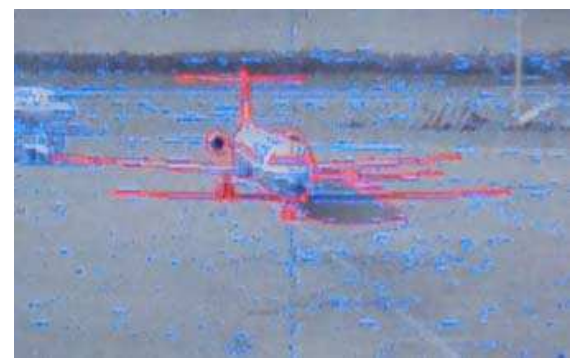

Fig.9. (b)

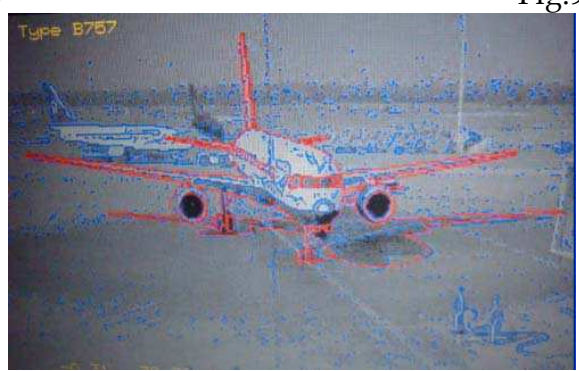

Fig.9. (c)

Fig. 9. (a) (b) (c) the proposed method images

\section{Conclusion}

In this paper, we presented an image segmentation algorithm based on adaptive weighted mathematical morphology edge detectors. The performance of the proposed algorithm has been demonstrated on the Lena image. The input of the proposed algorithm is a grey level image. The image was first processed by the mathematical morphological closing and 
dilation residue edge detector to enhance the edge features and sketch out the contour of the image, respectively. Then the adaptive weight SE operation was applied to the edgeextracted image to fuse edge gaps and hill up holds. Experimental results show it can not only primely extract detail edge, but also superbly preserve integer effect comparative to classical edge detection algorithm.

\section{References}

Lima, P.; Bonarini, A. \& Mataric, M. (2004). Name of Book in Italics, Publisher, ISBN, Place of Publication.

M. K. Kundu, B. B. Chaudhuri and D. Dutta Majumder, A parallel Graytone thinning algorithm (PGTA), Pattern Recognition. 12, 491- 496 (1991).

W. K. Pratt, Digital Image Processing, 2nd edn. Wiley, New York (1991).

SONG J, DELPE J. The analysis of morphological filters with multiple structuring elements [J]. Comp V is Graphics 7\& Image Proces, 1990,(50): 308 328.

Yao, Y. ACHARYA, R. and SRIHARI, S. "Image enhancement using mathematical morphology with adaptive structuring elements", nonlinear image processing, 1994, pp: 198-208(Vol. 2180 of SPIE Proceedings).

M. Pesaresi and J. A. Benediktsson, "A new approach for the morphological segmentation of high-resolution satellite imagery," IEEE Trans. Geosci. Remote Sensing, vol. 39, no. 2, pp. 309-320, 2001.

Heath M., Sarkar S., Sanocki T., and Bowyer K.: Comparison of Edge Detectors: A Methodology and Initial Study. Computer Vision and Image Understanding, 69(1): 38-54,1998.

Ziou D. and Tabbone S.: Edge Detection Techniques- An Overview, Technical report, No. 195, Dept Math \& Informatique. Universit de Sherbrooke ,1997.

M. H. Sedaaghi and Q. H. Wu, "Weighted morphological filter," Electronics Letters Online No:198, 2002.

P. Soille and H. Talbot, "Directional morphological filtering," IEEE Trans.Pattern Anal. Machine Intell, vol. 23, no.11, pp. 1313-1329, 2001.

R.V. Babu, K.R. Ramakrishnan, and S.H. Srinivasan, "Video Object Segmentation: Compressed Domain Approach," IEEE Transactions on Circuits and Systems for Video Technology, vol. 14, no. 4, pp. 462-474 ,April, 2004. 


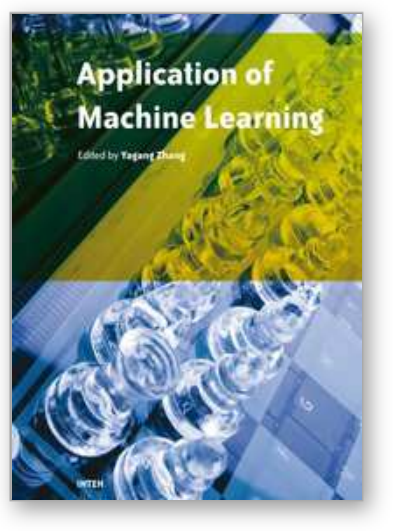

\section{Application of Machine Learning \\ Edited by Yagang Zhang}

ISBN 978-953-307-035-3

Hard cover, 280 pages

Publisher InTech

Published online 01, February, 2010

Published in print edition February, 2010

The goal of this book is to present the latest applications of machine learning, which mainly include: speech recognition, traffic and fault classification, surface quality prediction in laser machining, network security and bioinformatics, enterprise credit risk evaluation, and so on. This book will be of interest to industrial engineers and scientists as well as academics who wish to pursue machine learning. The book is intended for both graduate and postgraduate students in fields such as computer science, cybernetics, system sciences, engineering, statistics, and social sciences, and as a reference for software professionals and practitioners. The wide scope of the book provides them with a good introduction to many application researches of machine learning, and it is also the source of useful bibliographical information.

\section{How to reference}

In order to correctly reference this scholarly work, feel free to copy and paste the following:

Guo Yan-Ying, Yang Guo-Qing and Jiang Li-Hui (2010). Adaptive Weighted Morphology Detection Algorithm of Plane Object in Docking Guidance System, Application of Machine Learning, Yagang Zhang (Ed.), ISBN: 978953-307-035-3, InTech, Available from: http://www.intechopen.com/books/application-of-machinelearning/adaptive-weighted-morphology-detection-algorithm-of-plane-object-in-docking-guidance-system

\section{INTECH}

open science | open minds

\section{InTech Europe}

University Campus STeP Ri

Slavka Krautzeka 83/A

51000 Rijeka, Croatia

Phone: +385 (51) 770447

Fax: +385 (51) 686166

www.intechopen.com

\section{InTech China}

Unit 405, Office Block, Hotel Equatorial Shanghai

No.65, Yan An Road (West), Shanghai, 200040, China

中国上海市延安西路65号上海国际贵都大饭店办公楼 405 单元

Phone: +86-21-62489820

Fax: +86-21-62489821 
(C) 2010 The Author(s). Licensee IntechOpen. This chapter is distributed under the terms of the Creative Commons Attribution-NonCommercialShareAlike-3.0 License, which permits use, distribution and reproduction for non-commercial purposes, provided the original is properly cited and derivative works building on this content are distributed under the same license. 